\title{
Residual Stress Measurement of Laser-Engineered Net Shaping AISI 410 Thin Plates Using Neutron Diffraction
}

\begin{abstract}
P. PRATT, S.D. FELICELLI, L. WANG, and C.R. HUBBARD
In an attempt to relate the laser-engineered net shaping (LENS) process parameters, laser power and laser travel speed, to the quality of LENS-produced parts, strain measurements were taken at several predetermined points within seven LENS AISI 410 thin plates using the neutron diffraction method. The residual stresses at these points were then calculated using the measured strain values to ascertain how the internal stress varies as a function of the input parameters and location. It is found that the component of the stress in the vertical direction (i.e., perpendicular to the raster direction of the laser/powder nozzle) is dominant, in agreement with previous reports, and relatively insensitive to variations in process parameters. This was confirmed with numerical simulations performed with a thermomechanical model developed using the commercial program SYSWELD. The simulations also showed a good qualitative agreement with the measured simulated stresses.
\end{abstract}

DOI: $10.1007 / \mathrm{s} 11661-008-9660-9$

(c) The Minerals, Metals \& Materials Society and ASM International 2008

\section{INTRODUCTION}

PAST studies have employed different techniques for the measurement of internal residual stresses in parts manufactured by the laser-engineered net shaping (LENS) rapid fabrication process. $^{[1,2]}$ The results of these studies revealed high levels of type I macroscopic residual stress induced within the parts by the net shaping process. However, little is yet known of how the LENS input parameters affect the resulting levels and distributions of internal stresses. A more thorough understanding of the relationship between these parameters and residual stress could lead to better optimization of the LENS process and the production of higher quality components.

Using the method of neutron diffraction, strain measurements were taken at the High Flux Isotope Reactor Neutron Residual Stress Mapping Facility (NRSF2) at Oak Ridge National Laboratory for seven LENS-produced thin wall plates of stainless steel AISI 410. The dimensions of the plates were between 22- and $38-\mathrm{mm}$ wide and $15 \mathrm{~mm}$ in height, with a width of 1 to $3 \mathrm{~mm}$ depending on the laser power. One of these plates

P. PRATT, Graduate Student, Department of Mechanical Engineering, S.D. FELICELLI, Associate Professor, Department of Mechanical Engineering and Center for Advanced Vehicular Systems, and L. WANG, Postdoctoral Researcher, Center for Advanced Vehicular Systems, are with Mississippi State University, Mississippi State, MS 39762. Contact e-mail: felicelli@me.msstate.edu C.R. HUBBARD, Leader of Diffraction and Thermal Physical Properties Group, is with the Materials Science and Technology Division, Oak Ridge National Laboratory, Oak Ridge, TN 37831.

This article is based on a presentation given in the symposium entitled "Neutron and X-Ray Studies for Probing Materials Behavior," which occurred during the TMS Spring Meeting in New Orleans, LA, March 9-13, 2008, under the auspices of the National Science Foundation, TMS, the TMS Structural Materials Division, and the TMS Advanced Characterization, Testing, and Simulation Committee. Article published online October 15, 2008 is shown in Figure 1. The samples were produced at the facilities of Optomec (Albuquerque, NM). The plates were made by varying three process parameters: laser power, laser travel speed, and metal powder flow rate, according to Table I. The goal of the neutron measurements was to determine how the magnitude and distribution of residual stresses in the plates were affected by varying these input parameters.

\section{OVERVIEW: NEUTRON DIFFRACTION}

The neutron diffraction method for strain measurement at a reactor source uses a monochromatic beam of neutrons of known wavelength from a monochromator crystal. The atomic planes of certain crystallographic orientation $\{h k l\}$ diffract the neutrons at a scattering angle of $2 \theta$. Then, using Bragg's law of diffraction,

$$
\lambda=2 d_{h k l} \sin \theta
$$

where $\lambda$ is wavelength, $d_{h k l}$ is lattice spacing, and $\theta$ is diffraction angle.

The spacing between the lattice planes, $d_{h k l}$, was found by measurement of $2 \theta$. The diffracting lattice planes, $\{h k l\}$, were those with the plane normal parallel to the scattering vector, $\mathbf{Q}$, that is defined as

$$
\mathbf{Q}=\mathbf{q}_{\text {incident }}-\mathbf{q}_{\text {diffracted }}
$$

where $\mathbf{q}_{\text {incident }}$ and $\mathbf{q}_{\text {diffracted }}$ are the neutron beam vectors in the direction of the incoming and diffracted neutrons. ${ }^{[3]}$

For these measurements, a silicon monochromator with $\{330\}$ planes was used, diffracting neutrons with wavelength $\lambda=1.73 \AA$ that gave a scattering angle of $2 \theta=94.6 \mathrm{deg}$ from the sample. To identify the diffracting $\{h k l\}$, X-ray diffraction was performed on one of the sample plates and used to generate a plot of 


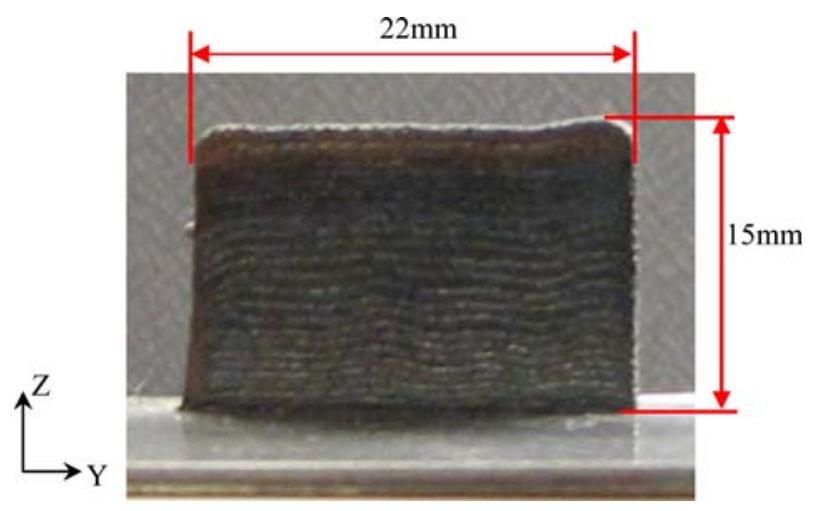

Fig. 1-LENS-produced thin-walled plate.

Table I. LENS Sample Process Parameters

\begin{tabular}{lccccc}
\hline & $\begin{array}{c}\text { Laser } \\
\text { Power } \\
\text { Number }\end{array}$ & $\begin{array}{c}\text { Number of } \\
\text { Layers }\end{array}$ & $\begin{array}{c}\text { Laser } \\
\text { Speed } \\
(\mathrm{mm} / \mathrm{s})\end{array}$ & $\begin{array}{c}\text { Sample } \\
\text { Length } \\
(\mathrm{mm})\end{array}$ & $\begin{array}{c}\text { Powder } \\
\text { Flow Rate } \\
\left(\mathrm{cm}^{3} / \mathrm{s}\right)\end{array}$ \\
\hline 1 & 300 & 25 & 2.54 & 38.1 & 37.85 \\
2 & 300 & 25 & 2.54 & 22.1 & 37.85 \\
3 & 300 & 25 & 4.23 & 25.4 & 44.16 \\
4 & 600 & 25 & 2.54 & 25.4 & 37.85 \\
5 & 600 & 25 & 4.23 & 25.4 & 44.16 \\
6 & 450 & 25 & 2.54 & 25.4 & 37.85 \\
7 & 450 & 25 & 4.23 & 25.4 & 50.47 \\
\hline
\end{tabular}

intensity vs $2 \theta$. The plot showed the $\{211\}$ planes to be diffracting for a $2 \theta$ of $94.6 \mathrm{deg}$.

\section{EXPERIMENTAL PROCEDURE}

Data was taken at prespecified locations within each sample. A total of 11 locations symmetrically arranged along the horizontal and vertical centerlines of the plates were selected, as shown in Figure 2. The boxes in Figure 2 represent gage volumes within the sample over which all the measured $d_{h k l}$ values are averaged. The dimensions of the gage volumes correspond to the cross section of the incident and diffracted neutron beams. The cross-sectional size was determined by the collimators through which the incident and diffracted neutrons pass, specifically, the areas of the rectangular collimator slits. The sizes of the gage volumes were necessarily quite small due to the geometry of the thin-walled plates. Table II lists the slit sizes used for each of the samples. The counting times were between 6 and 35 minutes per location.

The placement of the gage volumes is similar to that of a previous study of residual stress in LENS samples conducted by Rangaswamy et al. ${ }^{[2]}$ which was intended to show how stress varies as a function of position within the coordinate system of the part. However, the plates used in this study are 2 to $4 \mathrm{~mm}$ thinner than those measured by Rangaswamy et al., allowing for only one gage volume to be placed in the $X$ direction, as shown in Figure 2. By changing the orientation of the

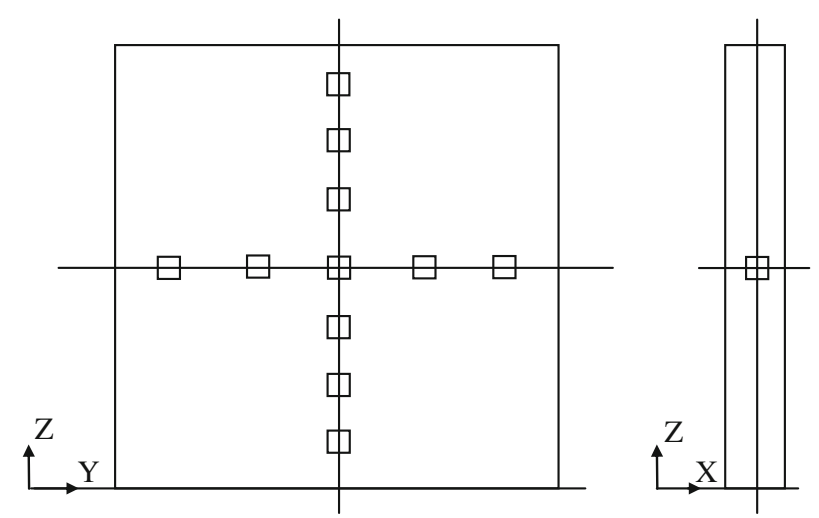

Fig. 2-Data sampling points within AISI 410 LENS plates.

Table II. Slit Sizes and Offsets Used for Diffraction Measurement

\begin{tabular}{lllllc}
\hline & \multicolumn{4}{c}{ Soller Slits } \\
\cline { 2 - 3 } & \multicolumn{2}{c}{ Incident } & & Diffracted \\
\cline { 2 - 3 } \cline { 5 - 6 } Sample & $\begin{array}{l}\text { Area } \\
\left(\mathrm{mm}^{2}\right)\end{array}$ & $\begin{array}{l}\text { Offset } \\
(\mathrm{mm})\end{array}$ & & $\begin{array}{l}\text { Width } \\
(\mathrm{mm})\end{array}$ & $\begin{array}{c}\text { Offset } \\
(\mathrm{mm})\end{array}$ \\
\hline $2,3,4,6,7$ & $0.7 \times 5$ & 30 & & 0.7 & 50 \\
1,5 & $0.7 \times 5$ & 50 & & 0.7 & 50 \\
\hline
\end{tabular}

sample with respect to the incoming neutron beam, the diffraction vector, $\mathbf{Q}$, was aligned with the three orthogonal axes of the part. Thus, $d_{h k l}$ could be measured for the $X, Y$, and $Z$ directions of the part coordinate system.

The gage volume was carefully embedded fully within the thin plate specimens by using neutron intensity measurements to accurately define the sample surface. The NRSF2 instrument uses seven detectors to collect the diffracted peak profile. These seven detectors are evenly spaced from approximately -15 to +15 deg out of the horizontal plane of diffraction. From data collected in these seven detectors, it was clear that the number of grains contributing to diffraction was low and the Debye ring was "spotty." To reduce this effect, an omega oscillation of the sample was employed to improve the grain statistics and to reduce the random errors in $d$-spacing measurements due to the spotty Debye ring. This oscillation was shown to improve, but not eliminate entirely, the random errors in $d_{h k l}$ measurement. Data from the seven detectors were corrected using corrections for detector intensity response, and for detector out-of-plane angle and offset, and then summed together before fitting a diffraction profile and determining the $2 \theta$, intensity, and full-width at halfmaximum.

To calculate strain, a small reference coupon, which had been cut from another LENS plate to relieve internal stresses, was measured along with each sample plate to obtain the value $d_{o}$, the measure of strain-free lattice spacing. Replicate measurements of the strainfree $d_{o}$ for each sample gave a range of root-meansquare deviation from the average of $5 \times 10^{-5} \AA$ to 
Table III. Polycrystalline $\{h k l\}$-Specific Elastic Constants for $\{211\}$ Reflection

$\{211\}$-Specific Elastic Constants of $\alpha \mathrm{Fe}$

$E_{211}$ *

$v_{211} *$

$225 \mathrm{GPa}$

Bulk Elastic Constants of AISI 410

$E_{\text {bulk }}$

vbulk

$215 \mathrm{GPa}$ 0.33

$4 \times 10^{-4} \AA$. The three orthogonal strain components were calculated from the measured $d_{h k l}$ components via Eq. [3]:

$$
\varepsilon_{i}=\frac{d_{i}-d_{o}}{d_{o}}
$$

where the index $i$ corresponds to $x, y$, and $z$.

Here, $d_{i}$ and $d_{o}$ are the lattice spacing, $d_{h k l}$, for the sample and the reference coupon, respectively, in the three orthogonal directions of the part coordinate system for crystallites with orientation $\{h k l\}$. Also, $\varepsilon_{i}$ represents the corresponding strain in these directions. Since the directions of the measured $d_{h k l}\left(\mathbf{d}_{x}, \mathbf{d}_{y}, \mathbf{d}_{z}\right)$ coincided with the coordinate axes of the thin-walled plates, the shear contributions to the strain tensor were negated and the calculated strain values $\varepsilon_{i}$ were taken as the principal strains. ${ }^{[4]}$

After calculating the strain with Eq. [3], the three orthogonal stress components were found using Hooke's law:

$$
\sigma_{i}=\frac{E_{h k l} \varepsilon_{i}}{\left(1+v_{h k l}\right)}+\frac{E_{h k l} v_{h k l}\left(\varepsilon_{i}+\varepsilon_{j}+\varepsilon_{k}\right)}{\left(1+v_{h k l}\right)\left(1-2 v_{h k l}\right)}
$$

where the indices $i, j$, and $k$ correspond to $x, y$, and $z$.

The diffraction elastic constants $E_{h k l}$ and $v_{h k l}$ in Eq. [4] are analogous to the bulk average Young's modulus and Poisson's ratio, respectively, but are specific to planes of a specific $\{h k l\}$ orientation. Due to the presence of numerous crystal orientations within a polycrystalline material, Kröner ${ }^{[5]}$ developed a model for predicting the elastic response in polycrystals composed of randomly oriented crystallites. Behnken and Hauk ${ }^{[6]}$ used the Kröner model to develop an algorithm for calculating polycrystal, $h \mathrm{kl}$ specific elastic moduli using the known values of the single-crystal compliance tensor of a material. This algorithm was used here to determine $E_{h k l}$ and $v_{h k l}$ in the $\{211\}$ direction for a polycrystal from the singlecrystal elastic constants of alpha iron obtained from Dever. ${ }^{[7]}$ The use of elastic constants intended for cases of random grain orientation is supported by the neutron data, because no significant variation in intensity was observed that would be indicative of significant textures, despite the \pm 4 deg omega oscillation. The calculated polycrystalline elastic constants, as well as the bulk elastic constants for AISI 410 are shown in Table III.

\section{MEASURED DATA AND DISCUSSION}

Previous research conducted by Rangaswamy et al..$^{[2]}$ on AISI 316 LENS plates revealed that the only

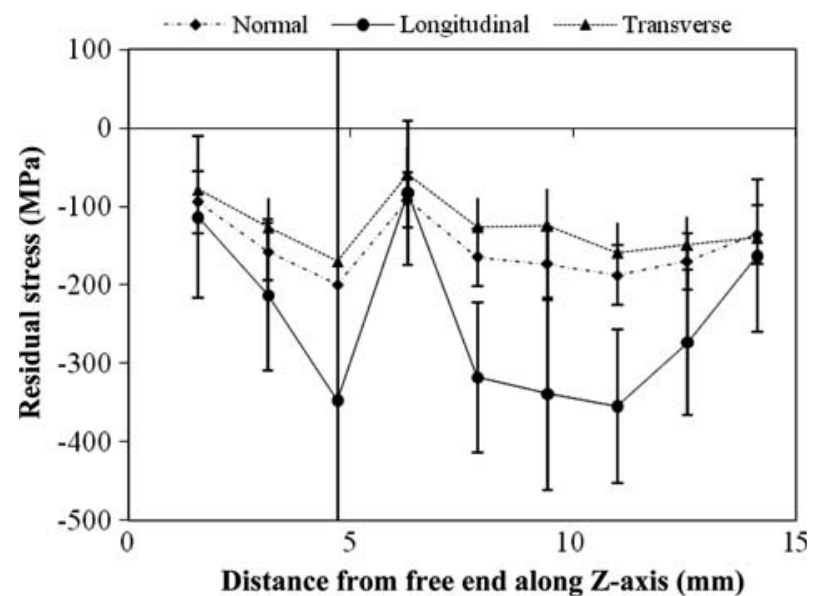

(a)

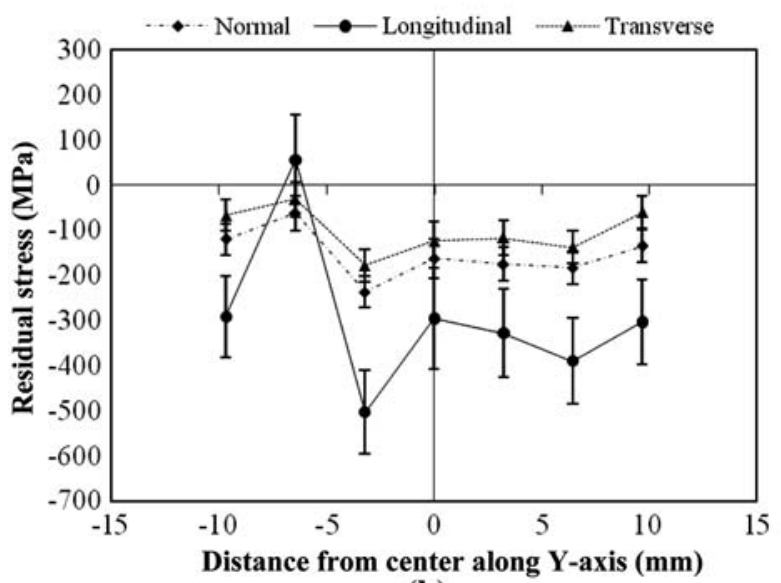

(b)

Fig. 3-Stress components as functions of position along (a) $Z$-axis of plate and (b) $Y$-axis of plate for sample 4 .

significant observed strain was that along the $Z$-axis of the part, which corresponds to the growth direction of the LENS build process. Such was also the case for residual stress measurements of welds conducted by Holden et $a l .{ }^{[8]}$ Initial findings in our measurement of strain in the $X$ and $Y$ directions for the AISI 410 samples clearly show the stress in the $Z$ direction to be dominant. Figure 3 shows the stress components $\sigma_{x}$ (normal), $\sigma_{y}$ (transverse), and $\sigma_{z}$ (longitudinal) of sample 4 plotted against position along the plate $Z$-axis in the center of the part, where the free end is the last deposited layer (i.e., the top surface of the plate). Based upon these findings and the available time period for neutron measurements, this analysis focused exclusively on the strain and stress in the $Z$ direction for all seven samples.

Figures 4 through 10 show how residual stress in the growth direction $\left(\sigma_{z}\right)$ changes as a function of position along the $Z$-axis and $Y$-axis of the LENS plates. For five of the samples (sample numbers 2, 3, 5, 6, and 7), seven data points were plotted along the plate $Z$-axis and five points along the plate $Y$-axis, with each data point corresponding to one of the gage volumes shown in Figure 2. However, for samples 1 and 4 , nine points were measured along the $Z$-axis and seven points along 


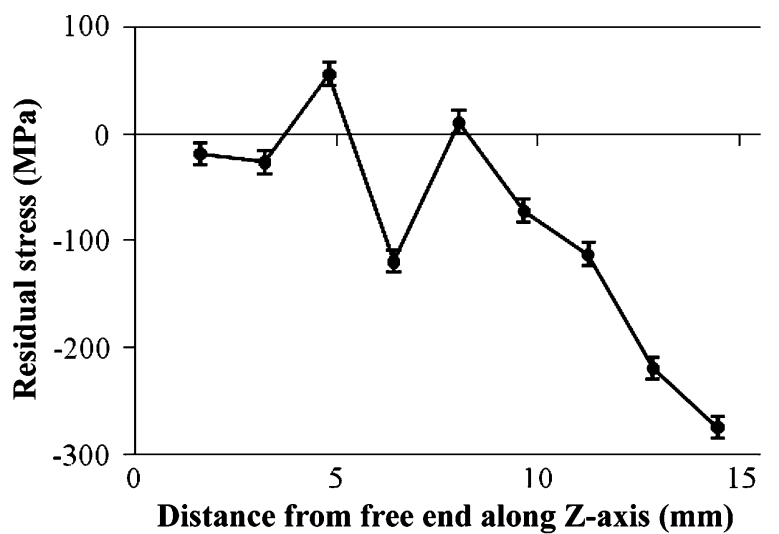

(a)

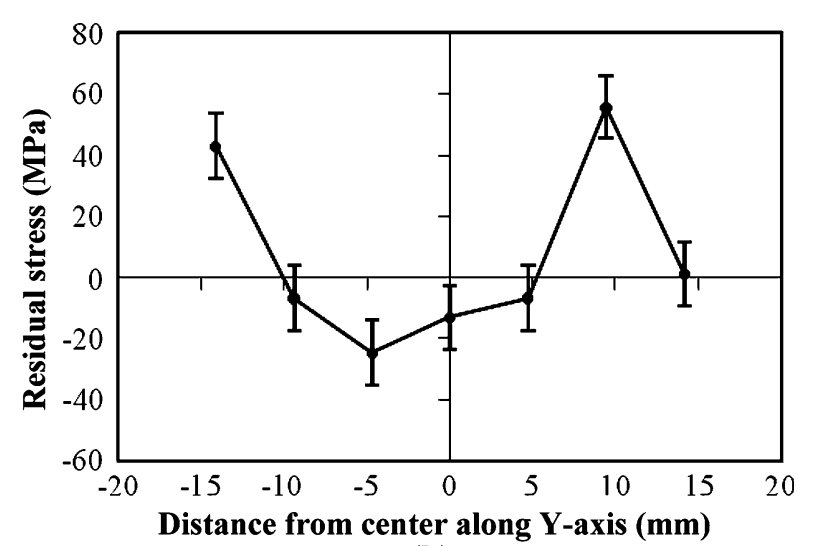

(b)

Fig. $4-\sigma_{z}$ as a function of position along (a) $Z$-axis of plate and (b) $Y$-axis of plate for sample 1 .

the $Y$-axis. The error bars shown in the figures are obtained by the propagation through Eqs. [1], [3], and [4] of random errors in $2 \theta$ and $d_{o}$ measurements. The variance in $2 \theta$ measurements was estimated by the NRSF2 peak fitting program, while that of $d_{o}$ was determined by replicate measurements on the reference coupon. Since the $d_{h k l}$ and $d_{o}$ values used for each sample were taken during the same experimental run and for the same set of diffracting planes, any systematic errors are removed in Eq. [3].

In Figures 3(a) and 7(a), which plot stress in sample 4 along the $Z$-axis, a very large error is calculated for the third gage volume from the free end. This error corresponds to a large value of variance in $2 \theta$ produced by the NRSF2 software at this location and may be due to the presence of a large grain within the gage volume. In this instance, the Debye cone would not cross the seven detectors uniformly, causing an artificial shift in the peak that leads to the unusually large $2 \theta$ variance.

Parts (a) of Figures 4 through 10 show that stress in the growth direction ( $Z$-axis) is mostly compressive for all of the measured samples. Within sample numbers 1 and 2, the stress becomes increasingly compressive along the $Z$-axis with increasing distance from the free end, while the stress profiles for samples 3, 4, 5, and 6 are more balanced, becoming highly compressive near the plate center and then leveling off closer to the substrate.

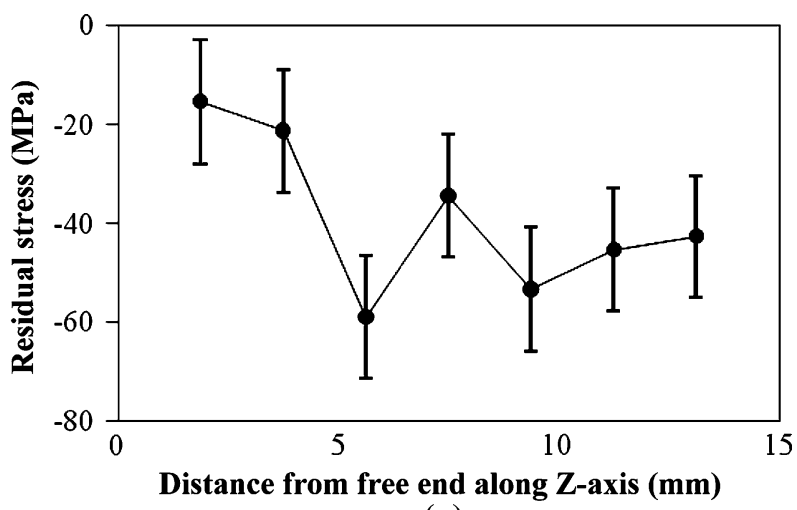

(a)

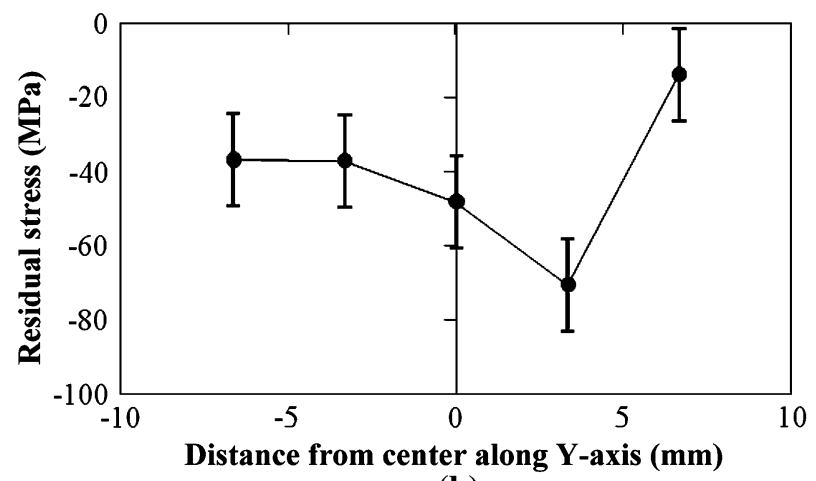

(b)

Fig. 5- $\sigma_{z}$ as a function of position along (a) $Z$-axis of plate and (b) $Y$-axis of plate for sample 2 .

Sample 7 shows the reverse trend of samples 1 and 2, becoming less compressive with increasing distance from the free end.

Parts (b) for Figures 4 through 10 show that the residual stresses along the plate width ( $Y$-axis) are mostly compressive near the center, becoming less compressive with increasing distance from the center. The stress at the center of sample 5 is slightly tensile, but a large uncertainty is associated with this location. Residual stresses at the plate edges must be tensile to satisfy a balance of forces; however, because the extremal gage volumes are 3 to $4 \mathrm{~mm}$ from the sample surfaces, this transition could not be measured for either direction. Also, the measurements are taken at the centerlines of the plate and do not include the plate front and back surface, where the stress could take tensile values and contribute to the overall force balance. Actually, Reference 16 shows that the stress is indeed tensile on most of the plate surface.

Pronounced peaks that deviate from the overall trends of the stress profiles are observed at certain locations within sample 1, 2, and 4 and can be seen in Figures 4(a), 4(b), 5(a), 7(a), and 7(b). To determine if these jumps are attributable to grain boundary effects, sample 4 was mounted and polished and electronbackscattered diffraction (EBSD) analysis performed on a $0.3 \mathrm{~mm} \times 0.3 \mathrm{~mm}$ section using a field-emission gun scanning electron microscope (FEG-SEM). The results, shown in Figure 11, reveal a fine grain structure with approximately 90 pct of the grains being less than 


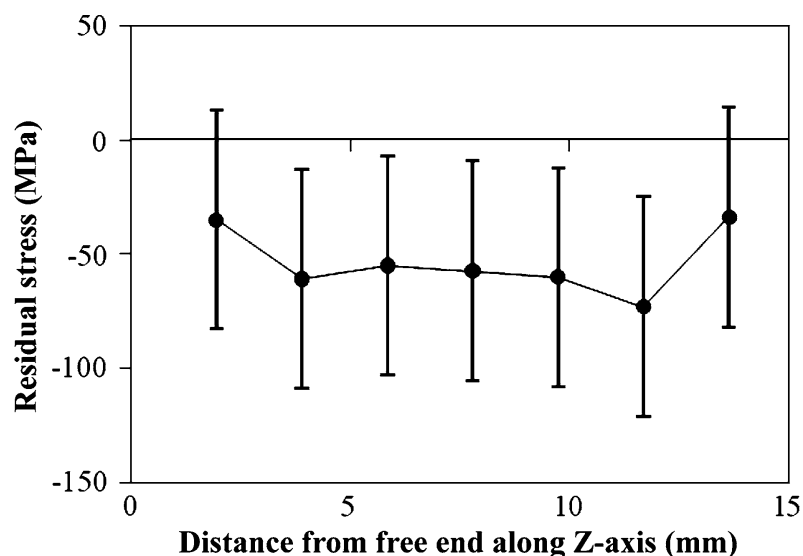

(a)

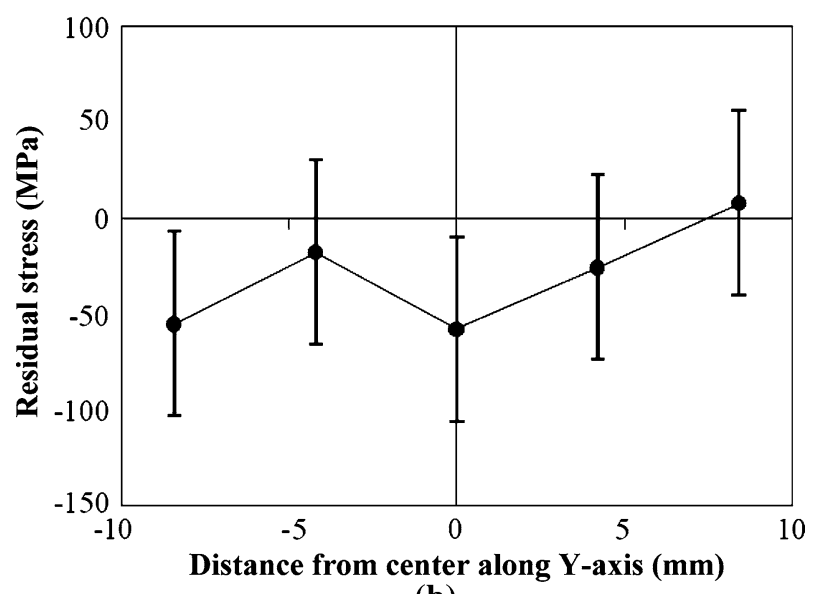

(b)

Fig. $6-\sigma_{z}$ as a function of position along (a) $Z$-axis of plate and $(b)$ $Y$-axis of plate for sample 3 .

$10 \mu \mathrm{m}^{2}$ in size, which are quite small in comparison to the cross section of the incoming beam $(0.7 \mathrm{~mm} \times$ $5 \mathrm{~mm}$ ). Additionally, a review of the neutron data shows that intensities measured by the seven detectors remain consistent at all gage volumes in the samples. Accordingly, the observed jumps in strain/stress are likely not due to grain boundary effects. However, because all seven detectors measured variations in $2 \theta$ at these locations as compared with the neighboring gage volumes, the anomalies almost certainly originate in the material and are not due to random instrumentation error.

A metallographic analysis performed on another mounted and polished plate sample revealed large, irregularly shaped defects that appear to be pores. These defects, which are shown in Figure 12, were found to be larger than $200 \mu \mathrm{m}$ in length and could affect the averaging of $d_{h k l}$ within the gage volumes. The observed jumps may be attributable to these defects.

Table IV summarizes the maximum and average values of the stress (which are compressive) obtained from all of the measured stress values for each one of the samples. An analysis of the data in Table IV reveals that no definite trend can be established between the maximum stress measured in the sample and the process parameters of laser power and travel speed. In other

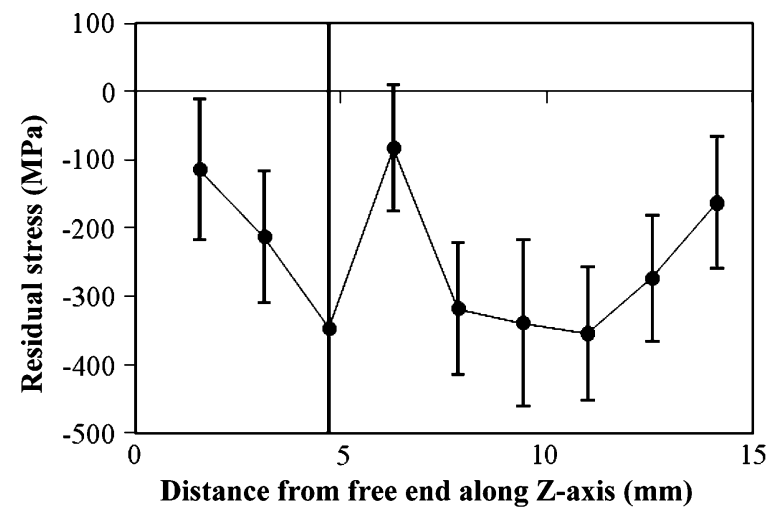

(a)

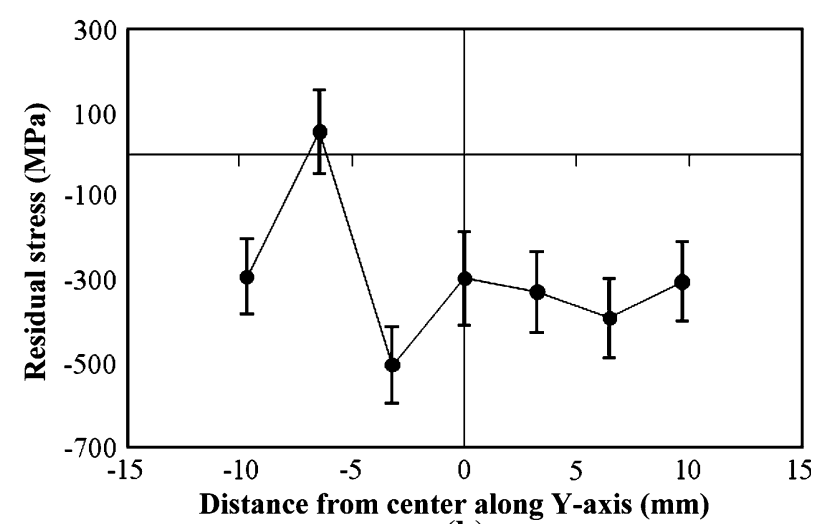

(b)

Fig. 7- $\sigma_{z}$ as a function of position along $(a) Z$-axis of plate and (b) $Y$-axis of plate for sample 4 .

words, for the range of parameters studied, there is no evidence that the maximum residual stress in the sample can be reduced by adjusting the power or travel speed of the laser. When looking at average values, there seems to be a trend to overall lower values of the stress for lower laser powers. In order to contrast this observation against an independent method of analysis, a numerical model of the process was developed and used to perform simulations for the geometry and parameters of the experimental study. The results of this analysis are described next.

\section{COMPARISON WITH NUMERICAL SIMULATIONS}

A three-dimensional thermomechanical model of the LENS process was developed using the commercial program SYSWELD. ${ }^{[9]}$ The model is based on the thermal model presented in References 10 through 14, which was validated with experimental temperature measurement of an actual deposition process. ${ }^{[15]}$ This model was used to predict the temperature distribution and cooling rate for the LENS deposition of a thinwalled structure (plate) of AISI 410 stainless steel. A mechanical model based on SYSWELD's material library was coupled to the thermal formulation in order to calculate deformation and stress during deposition as well as the final residual stress of the built plate. 


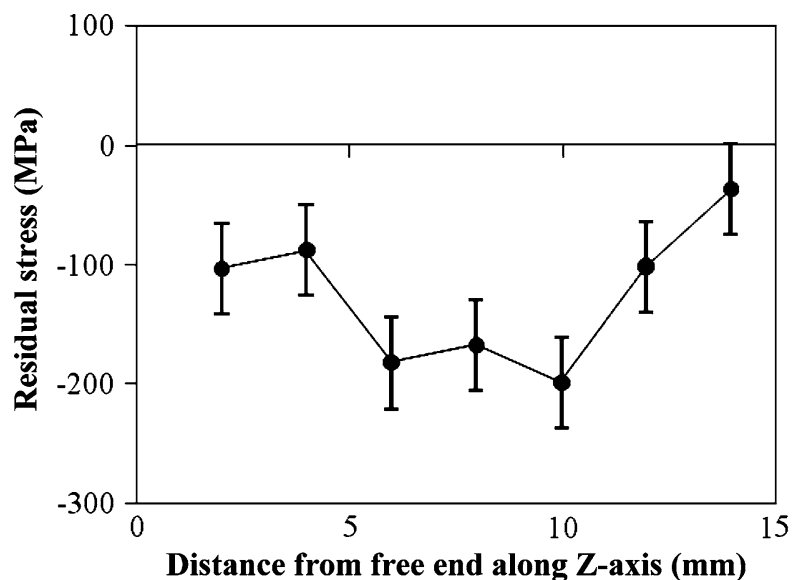

(a)

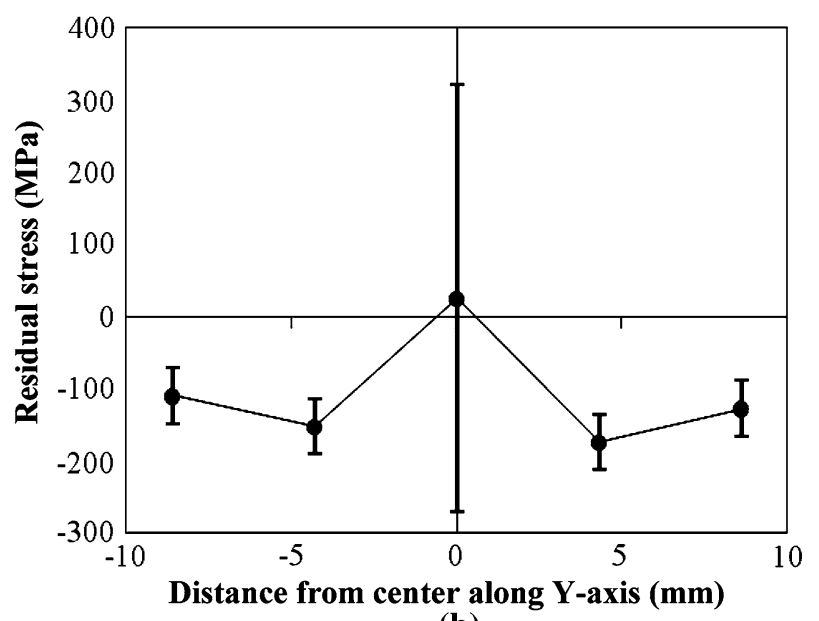

(b)

Fig. $8-\sigma_{z}$ as a function of position along $(a) Z$-axis of plate and $(b)$ $Y$-axis of plate for sample 5 .

The mechanical model uses an additive constitutive relation for strains due to elastic, thermal, and plastic deformation, including transformation-induced plasticity. Details of the development and implementation of the mechanical model are reported elsewhere. ${ }^{[16]}$

The geometry and finite element mesh used for the simulations are shown in Figure 13. The structure was built by overlapping 10 single tracks of material, each with a length of $10.0 \mathrm{~mm}$, a thickness of $0.5 \mathrm{~mm}$, and a width of $1.0 \mathrm{~mm}$. This produced a plate $5-\mathrm{mm}$ tall. The plate was fabricated on the surface of a substrate that was 1-mm thick, 4-mm wide, and 10-mm long. Although smaller than the actual plates, the model configuration serves well the purpose of illustrating the sensitivity of stress to process parameters, and the modeling results will compare with the experimental measurements in a qualitative way, in addition to saving considerable computational time. The three-dimensional thermomechanical simulations are computationally rather costly, with each ten-layer deposition calculation taking more than a day of computer time in a medium fast workstation.

The laser beam moves in the same direction $(+Y$ direction) for each pass. A dense mesh was used for the

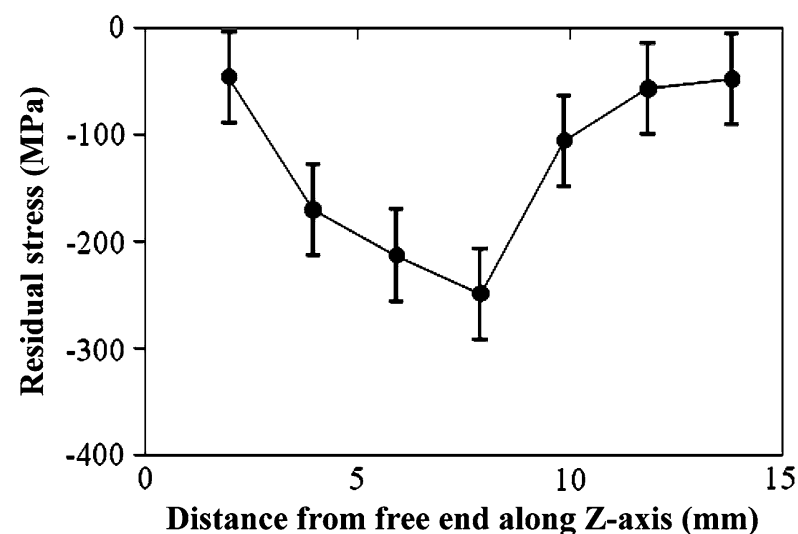

(a)

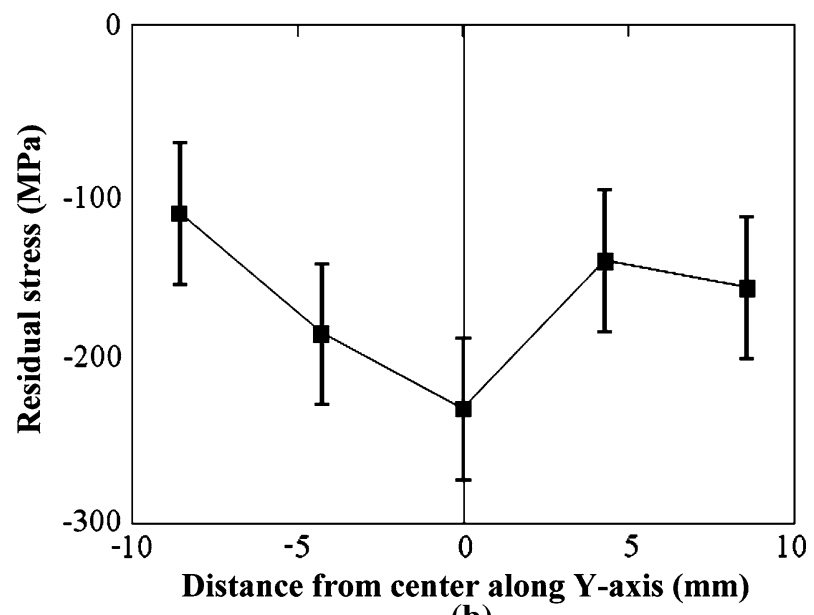

(b)

Fig. $9-\sigma_{z}$ as a function of position along $(a) Z$-axis of plate and $(b)$ $Y$-axis of plate for sample 6 .

plate and the contact area with the substrate, where higher thermal gradients are expected. In order to compensate for the heat-sink effect of the substrate, an optimized laser power program, as described in Reference 12, was employed to achieve a steady size of the molten pool during deposition. For the three values of travel speed simulated $(2.5,4.2$, and $8.5 \mathrm{~mm} / \mathrm{s})$, this required average laser powers of 254,285 , and $344 \mathrm{~W}$, respectively.

Figure 14 shows the simulated distribution of residual stress $\sigma_{z}$ after the plate has cooled to room temperature, for the three values of the laser travel speed. Figure 14(a) shows the stress profile along the laser travel direction for the third deposited layer, while in Figure 14(b), the profile corresponds to the vertical center line along the plate measured from the free end toward the substrate. The stress values in individual finite element cells are indicated by points of different colors and shapes to differentiate the three travel speeds. Fitting curves for each travel speed are also indicated, as well as an average value of the overall stress level shown as a horizontal dashed line in Figure 14(a).

The stress profiles shown in Figure 14 follow the same trends observed in the experiments. In the horizontal direction, a compressive stress in the center region of the 


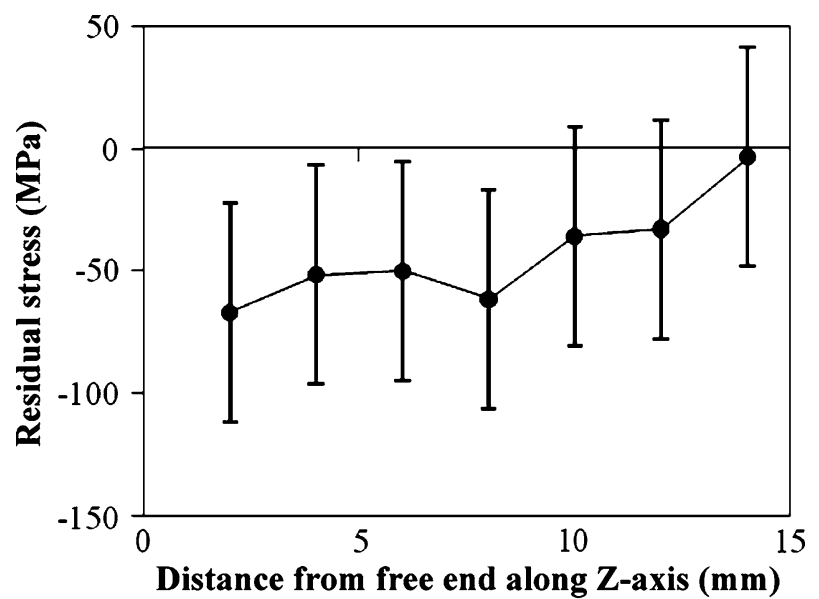

(a)

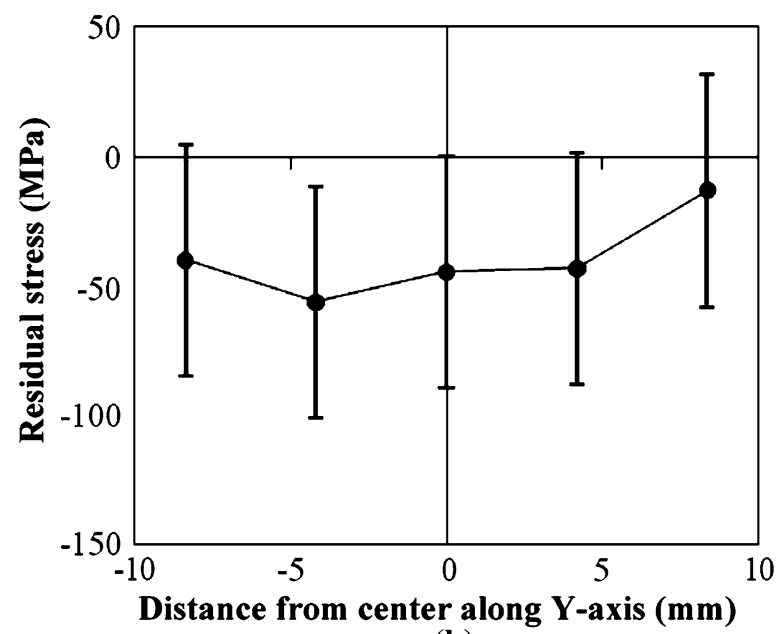

(b)

Fig. $10-\sigma_{z}$ as a function of position along (a) $Z$-axis of plate and (b) $Y$-axis of plate for sample 7 .

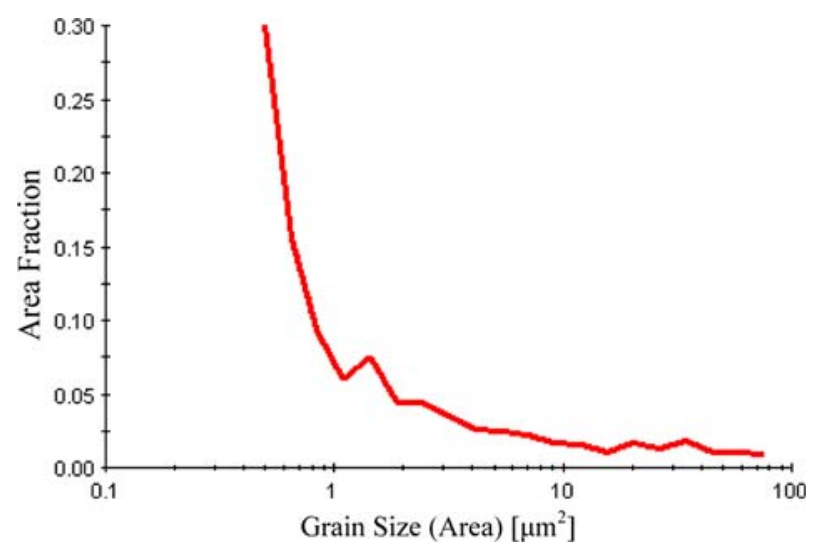

Fig. 11-Area fraction of grains of different sizes from plate sample 4, obtained by EBSD analysis.

plate is observed, shifting to tensile or less compressive values near the side edges, which has the same trends as Figures 4(b) and 7(b). Along the vertical direction, the compressive magnitude of the stress increases with distance from the top free end and becomes less

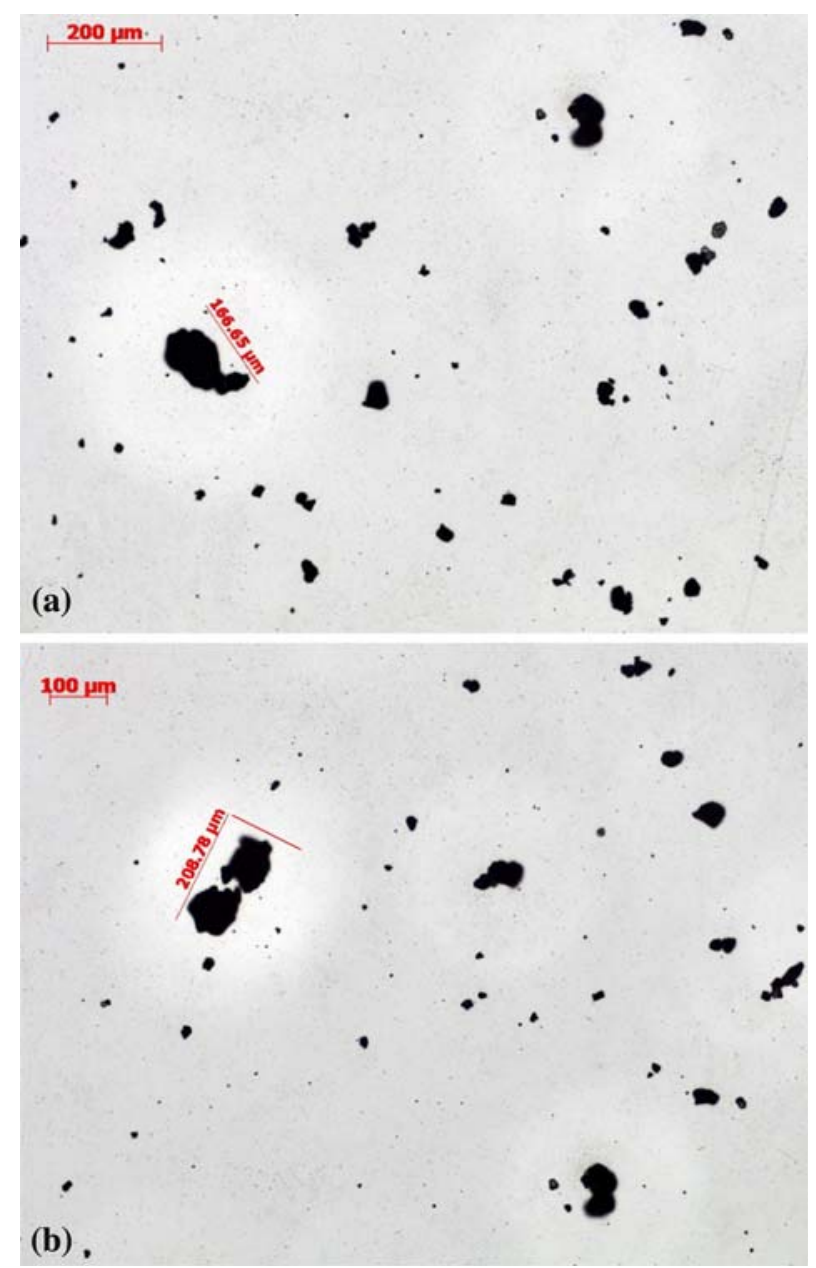

Fig. 12-(a) and (b) Porosity observed in AISI 410 plate with optical microscopy at 5 times magnification.

Table IV. Maximum and Average Compressive Stress in Samples

\begin{tabular}{lcccc}
\hline Sample & $\begin{array}{c}\text { Maximum } \\
\text { Stress } \\
(\mathrm{MPa})\end{array}$ & $\begin{array}{c}\text { Average } \\
\text { Stress } \\
(\mathrm{MPa})\end{array}$ & $\begin{array}{c}\text { Laser } \\
\text { Power } \\
(\mathrm{W})\end{array}$ & $\begin{array}{c}\text { Laser } \\
\text { Speed } \\
(\mathrm{mm} / \mathrm{s})\end{array}$ \\
\hline 1 & -379.75 & -151.43 & 300 & 2.5 \\
2 & -70.65 & -39.88 & 300 & 2.5 \\
3 & -73.08 & -43.77 & 300 & 4.2 \\
4 & -503.16 & -266.13 & 600 & 2.5 \\
5 & -199.14 & -118.31 & 600 & 4.2 \\
6 & -248.69 & -143.35 & 450 & 2.5 \\
7 & -67.00 & -41.78 & 450 & 4.2 \\
\hline
\end{tabular}

compressive at locations close to the substrate, which has the same trends as Figures 6(a) and 7(a). Note that there is a small increase in the center of the stress profile along the horizontal direction (Figure 14(a)), something also observed in some of the measured samples (Figures 5(b) and 6(b)) and attributed to the presence of pores or grain boundaries. Since no such defects are included in the model, there may also exist a thermal contribution to these apparently anomalous variations in the measured stress profiles. From the 


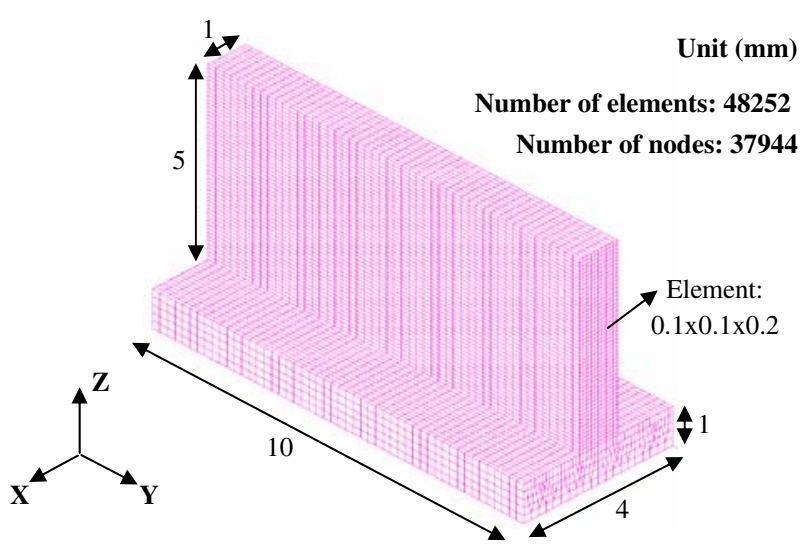

Fig. 13 - Geometry and meshing of thin plate and substrate used for the thermomechanical model.

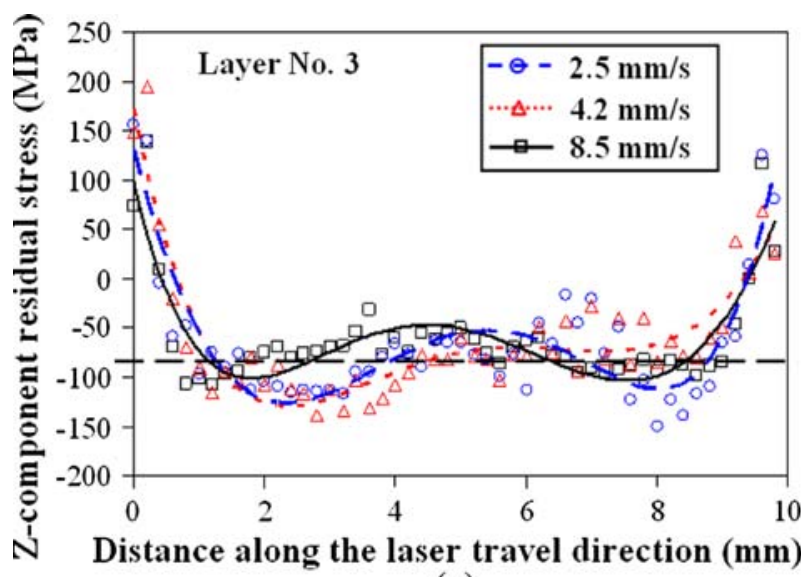

(a)

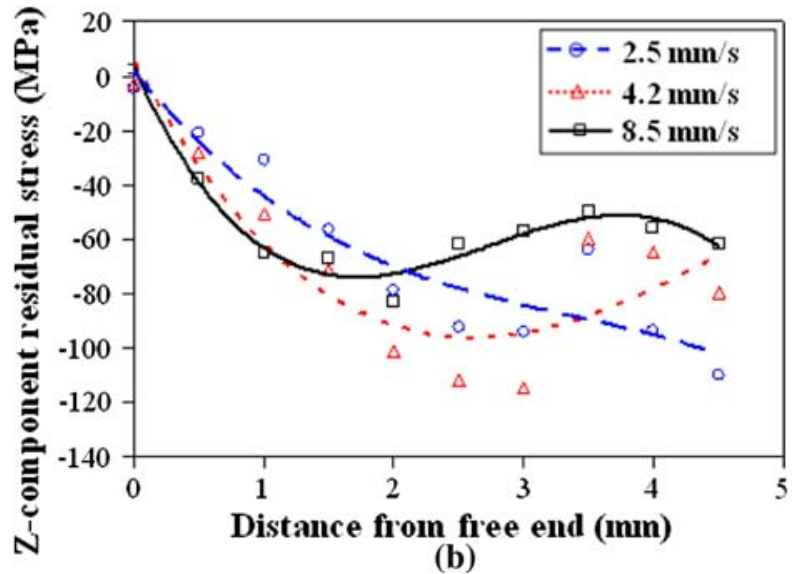

Fig. $14-Z$-component residual stress along the $(a)$ travel direction and $(b)$ vertical direction as a function of laser travel speed.

highly intertwined distribution of stress points corresponding to different travel speeds and the stress profile curves shown in Figure 14, there seems to be no definite trend of the variation of this component of the stress with laser travel speed, an observation also made from the analysis of the experimental results.

A qualitative comparison between simulated results and measured data is given in Figure 15, which shows

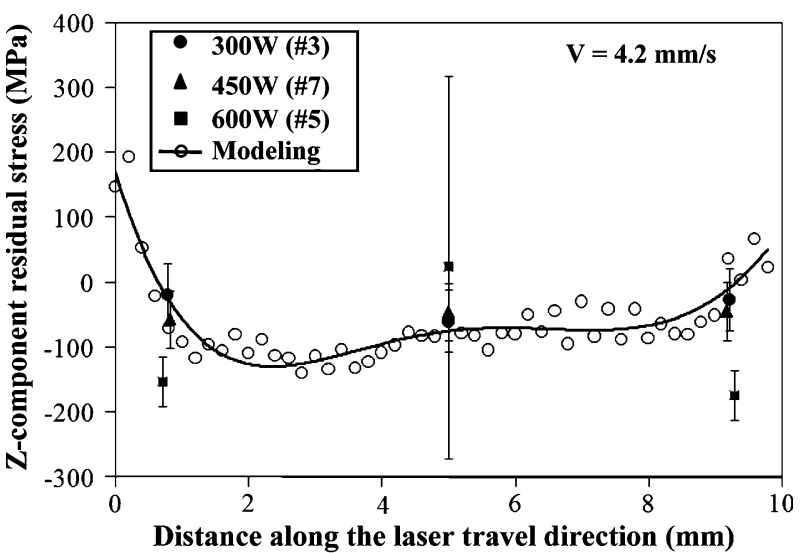

(a)

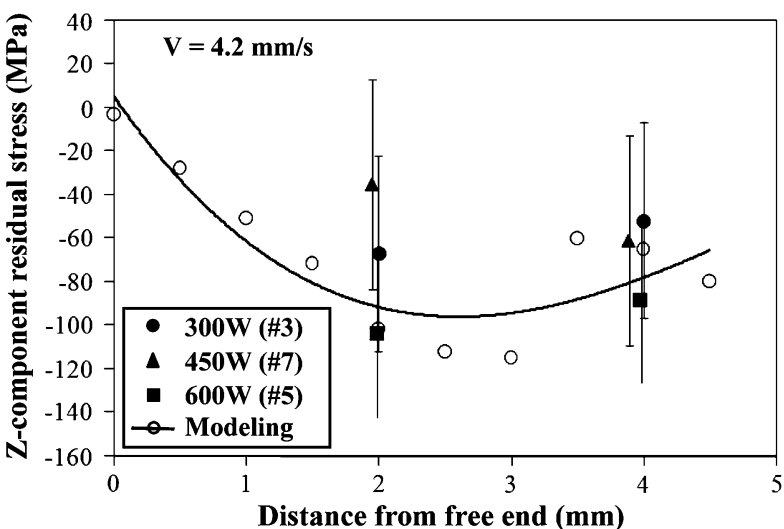

(b)

Fig. 15-Simulated and measured Z-component residual stress along the $(a)$ travel direction and $(b)$ vertical direction as a function of laser power. Modeling results correspond to an average laser power of $285 \mathrm{~W}$.

stress profiles for a laser travel speed of $4.2 \mathrm{~mm} / \mathrm{s}$. The solid line is a fitting curve of simulated stress values (hollow circular points), while the solid points are measured residual stresses for different levels of the laser power. Because the real samples are taller and wider than the simulated model sample, the horizontal profile comparison is done for the first $5 \mathrm{~mm}$ measured from the vertical centerline, while the vertical profile comparison is done for the first $5 \mathrm{~mm}$ measured from the free end. This limits the comparison to 3 or 4 measured data points for each profile.

It can be observed that the simulated profiles for both the horizontal direction (Figure 15(a)) and vertical direction (Figure 15(b)) agree rather well with the measured data corresponding to a laser power of $300 \mathrm{~W}$ (sample 3), which is close to the average value of $285 \mathrm{~W}$ used in the simulations through the optimized laser power program. This is a noticeable agreement, considering the several simplifications used in the construction of the model. In particular, it seems to indicate that the constitutive model used for the material performs satisfactorily in predicting the overall level of the $\sigma_{z}$ residual stress. However, more experimental validations in a wider range of process parameters would be necessary to confirm this observation. 


\section{CONCLUSIONS}

The residual stress of several AISI 410 thin plates built with the LENS process under different process parameters was measured with the method of neutron diffraction. In agreement with previous reports, the component of the residual stress along the growth axis was found to be compressive in most of the parts and dominant with respect to the other components. The stress changes considerably across the plate. The maximum value of the stress varied between 5 and 30 pct of the yield point. Surprisingly, the magnitude of the stress showed no significant trend with the laser travel speed and only a slight trend to increase with the value of the laser power. A thermomechanical model was developed to simulate the process and complement the experimental data. The simulation results compare well with the measured stress and confirm some of the experimental findings. This study quantified the effect of two of the most important LENS parameters, laser power and travel speed, on the residual stress level of built parts, using a model and neutron diffraction stress measurements.

\section{ACKNOWLEDGMENTS}

The authors appreciate the sponsorship of the United States Army TACOM and the Center of Advanced Vehicular Systems (CAVS) at Mississippi State University (MSU). Helpful discussions with Professor John Berry, Department of Mechanical Engineering, MSU, and Dr. Haitham El Kadiri, CAVS, are also gratefully acknowledged. The neutron diffraction measurements were done within the High Temperature Materials Laboratory User Program, which is sponsored by the Assistant Secretary for Energy Efficiency and Renewable Energy, Office of FreedomCAR, and
Vehicle Technologies, as part of the High Temperature Materials Laboratory User Program, Oak Ridge National Laboratory, managed by UT-Battelle, LLC, for the United States Department of Energy under Contract No. DE-AC05-00OR22725.

\section{REFERENCES}

1. M.L. Griffith, M.E. Schlienger, L.D. Harwell, M.S. Oliver, M.D. Baldwin, M.T. Ensz, J.E. Smugeresky, M. Essien, J. Brooks, C.V. Robino, W.H. Hofmeister, M.J. Wert, and D.V. Nelson: Mater. Des., 1999, p. 107.

2. P. Rangaswamy, M.L. Griffith, M.B. Prime, T.M. Holden, R.B. Rogge, J.M. Edwards, and R.J. Sebring: Mater. Sci. Eng. A, vol. 399 , pp. $72-83$.

3. A.J. Allen, M.T. Hutchings, and C.G. Windsor: Adv. Phys., 1985, vol. 34 , pp. $446-47$

4. R.A. Winholtz: in Analysis of Residual Stress by Diffraction Using Neutron and Synchrotron Radiation, M.E. Fitzpatrick and A. Lodini, eds., Taylor and Francis, New York, 2003, pp. 60-77.

5. E. Kröner: Z. Phys., 1958, vol. 5, pp. 504-18.

6. H. Behnken and V. Hauk: Z. Metallkd., 1985, vol. 77, pp. 620-25.

7. D.J. Dever: J. Appl. Phys., 1972, vol. 43, p. 3293.

8. T.M. Holden, H. Suzuki, D.G. Carr, M.I. Ripley, and B. Clausen: Mater. Sci. Eng. A, 2006, vol. 437, pp. 33-37.

9. SYSWELD Reference Manual, ESI Group, Paris, 2005.

10. L. Wang and S.D. Felicelli: Mater. Sci. Eng. A, 2006, vols. 435 436, pp. 625-31

11. L. Wang and S.D. Felicelli: J. Manuf. Sci. Eng., 2007, vol. 129, pp. $1028-34$.

12. L. Wang, S.D. Felicelli, Y. Gooroochurn, P.T. Wang, and M.F. Horstemeyer: Mater. Sci. Eng. A, 2008, vol. 474, pp. 148-56.

13. L. Wang and S. Felicelli: in Materials Processing Fundamentals, P. Anyalebechi, ed., TMS, Warrendale, PA, 2007, pp. 63-72, IBN: 978-0-87339-674-5.

14. L. Wang, S. Felicelli, Y. Gooroochurn, P.T. Wang, and M.F. Horstemeyer: 2006, Proc. SFF 2006-1 $17^{\text {th }}$ Solid Freeform Fabrication Symp., University of Texas at Austin, Austin, TX, Aug. 2006, pp. 453-63.

15. L. Wang, S. Felicelli, and J. Craig: Proc. SFF 2007-18th Solid Freeform Fabrication Symp., University of Texas at Austin, Austin, TX, Aug. 6-8, 2007, pp. 100-11.

16. L. Wang, S.D. Felicelli, and P. Pratt: Mater. Sci. Eng. A, 2008, vol. 496 , pp. $10-20$ 